\title{
Aging in the Large CDF Axial Drift Chamber
}

\author{
D. Allspach, D. Ambrose, M. Binkley, C. Bromberg, K. Burkett, R. Kephart, R. Madrak, T. Miao, \\ A. Mukherjee, R. Roser, R.L. Wagner
}

\begin{abstract}
The Central Outer Tracker (COT) is a large axial drift chamber in the Collider Detector at Fermilab operating with a gas mixture that is 50/50 argon/ethane with an admixture of $1.7 \%$ isopropanol. In its first two years of operation the COT showed unexpected aging with the worst parts of the chamber experiencing a gain loss of $\sim 50 \%$ for an accumulated charge of $\sim 35 \mathrm{mC} / \mathrm{cm}$. By monitoring the pulse height of hits on good tracks, it was possible to determine the gain as a function of time and location in the chamber. In addition, the currents of the high voltage supplies gave another monitor of chamber gain and its dependence on the charge deposition rate. The aging was worse on the exhaust end of the chamber consistent with polymer buildup as the gas flows through the chamber. The distribution in azimuth suggests that aging is enhanced at lower temperatures, but other factors such as gas flow patterns may be involved. Elemental and molecular analysis of the sense wires found a coating that is mostly carbon and hydrogen with a small amount of oxygen; no silicon or other contaminants were identified. High resolution electron microscope pictures of the wire surface show that the coating is smooth with small sub-micron nodules. In the course of working with the chamber gas system, we discovered a small amount of $\mathrm{O}_{2}$ is enough to reverse the aging. Operating the chamber with $\sim 100 \mathrm{ppm}$ of $\mathrm{O}_{2}$ reversed almost two years of gain loss in less than 10 days while accumulating $\leq 2 \mathrm{mC} / \mathrm{cm}$.
\end{abstract}

\section{INTRODUCTION}

The Collider Detector at Fermilab (CDF)[1] is a large magnetic detector built to study $2 \mathrm{TeV}$ proton anti-proton collisions at the Fermilab Tevatron Collider. At its center is the Central Outer Tracker (COT)[2], a large cylindrical tracking chamber coaxial with the beam that measures the 3 momentum of charged particles with excellent resolution.

This paper discusses the aging observed in the COT and our efforts to remedy it. Section II reviews the technical details of the chamber. Section III describes the definitive evidence for aging in the chamber. Section IV discusses the nature of the aging seen in the COT and our initial ideas for restoring chamber gain. Section $\mathrm{V}$ describes the steps we took and how they led to adding oxygen to reverse the aging in

Manuscript received November 10, 2004. This work was supported by the U. S. Department of Energy.

D. Allspach, D. Ambrose, M. Binkley, K. Burkett, R. Kephart, R. Madrak, T. Miao, A. Mukherjee, R. Roser, and R.L. Wagner are with Fermi National Accelerator Laboratory, P.O. Box 500, Batavia, IL USA (telephone: 630-8403000; e-mail: allspach@fnal.gov; ambrose@fnal.gov; binkley@fnal.gov; burkett@fnal.gov; kephart@fnal.gov; madrak@fnal.gov; tmiao@fnal.gov; mukherjee@fnal.gov; roser@fnal.gov; wagner@fnal.gov ).

C. Bromberg is at Fermi National Accelerator Laboratory on leave from Michigan State University, Physics Dept., 3225 BPS Building, East Lancing, Michigan 48824 USA (email: Bromberg@pa.msu.edu). the COT. Section VI reviews some of the tests and studies we made to get a better understanding of the aging. Section VII presents the conclusions.

\section{DETAILS OF THE COT}

The COT resides inside a $1.4 \mathrm{~T}$ solenoid magnet in the middle of the CDF detector. It is a cylinder that measures approximately $3 \mathrm{~m}$ long by $3 \mathrm{~m}$ in diameter. There are more than 30,000 sense wires and 40,000 potential wires strung between two thick aluminum endplates. The wires are arranged radially in 8 annular regions called super-layers (4 axial and 4 small-angle stereo). Inside each super-layer the wires are organized in a jet cell configuration with each cell tilted $35^{\circ}$ with respect to the radial direction. The variable in the axial direction is $\mathrm{z}$ with the chamber center at $\mathrm{z}=0$. The azimuthal variable is phi which has a correspondence with the super-layer cell number.

There are 12 sense wire layers per cell giving a total of 96 sense wire layers in the 8 super-layers. Between each pair of sense wires and at the end of the cell are "potential" wires that improve the shape of the drift field. Both sense and potential wires are $40 \mu \mathrm{m}$ gold plated tungsten and the cathodes are vapor-deposited gold on $6.35 \mu \mathrm{m}$ mylar foils. The maximum drift distance is $0.9 \mathrm{~cm}$. The sense wires are operated at $\sim 3000$ $\mathrm{V}$ and the potential wires at $\sim 1600 \mathrm{~V}$ with the cathode planes at ground. The gas gain is $\sim 20000$.

The chamber gas is argon/ethane (50/50) with an admixture of $1.7 \%$ ispropanol. The gas is injected through one endplate and exhausted through the other endplate. The total flow is adjusted to be approximately equal for each super-layer with a uniform distribution in phi. The flow was from negative to positive $\mathrm{z}$ during almost all of the aging. Initially the gas flow was $20 \mathrm{SCFH}$ which corresponds to one volume exchange every 30 hours. Test monitor chambers saw no signs of aging.

The thick aluminum endplates are kept at about $70^{\circ} \mathrm{F}$ by a SUVA cooling system. Due to heat from another detector system, the outer cylinder of the chamber is warmer, varying from about $74^{\circ} \mathrm{F}$ at the bottom to $77.5^{\circ} \mathrm{F}$ at the top. The inner cylinder temperature variation is larger due to the heat generated by the silicon detector and the heat removed by its cooling system; the temperature varies from about $69^{\circ} \mathrm{F}$ on the bottom to $78^{\circ} \mathrm{F}$ on the top. Temperature variations within a store are always less than $2^{\circ} \mathrm{F}$.

There are several features built into the system to help monitor the gain of the COT. In order to facilitate $\mathrm{dE} / \mathrm{dx}$ 
measurements in the physics data, the amplifier-shaperdiscriminator sets its output pulse width proportional to the integrated input charge. The width of all hits, and thereby the pulse-height, are stored in the CDF physics data sets allowing a determination of the gain as a function of position in the chamber. In addition all the high voltages and currents are archived every $\sim 10$ minutes. Archives are also kept of the Tevatron luminosity and some of the loss monitor rates.

\section{EVIDENCE FOR AGING}

Despite the mechanisms in place to monitor chamber gain, detecting aging was not easy. There were hints early in 2003 and through the summer. The online track trigger which has limited redundancy exhibited phi dependent inefficiencies. Definitive evidence of aging came from careful studies of the offline tracking in November-December 2003. They showed that the gain was reduced in the inner super-layers relative to the outer super-layers and the gain was reduced at the exhaust end of the chamber relative to the input. Fig. 1 shows the gain

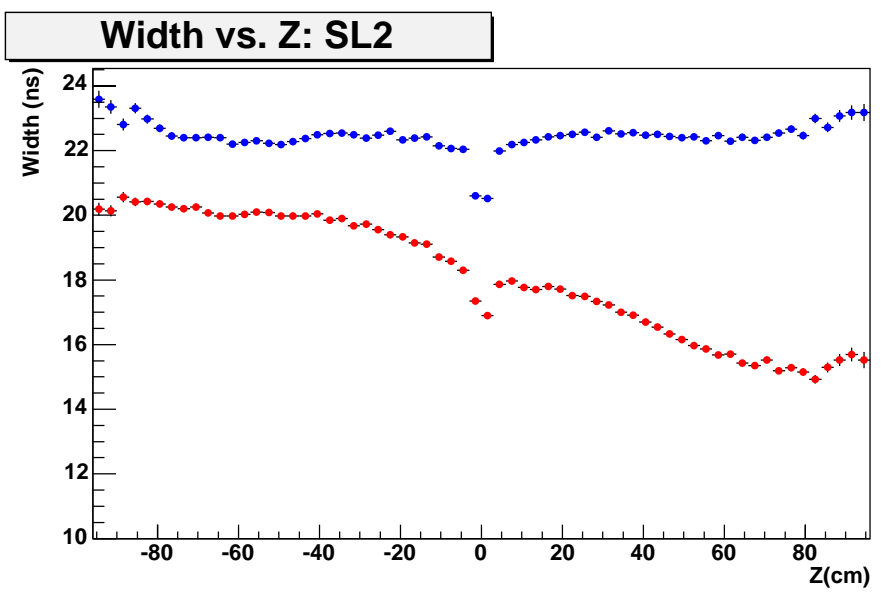

Fig. 1. Average pulse width, which is proportional to the gain, versus distance $\mathrm{z}$ along the wires. Blue points are from an early run with no gain loss and red points are from a latter run with considerable gain loss.

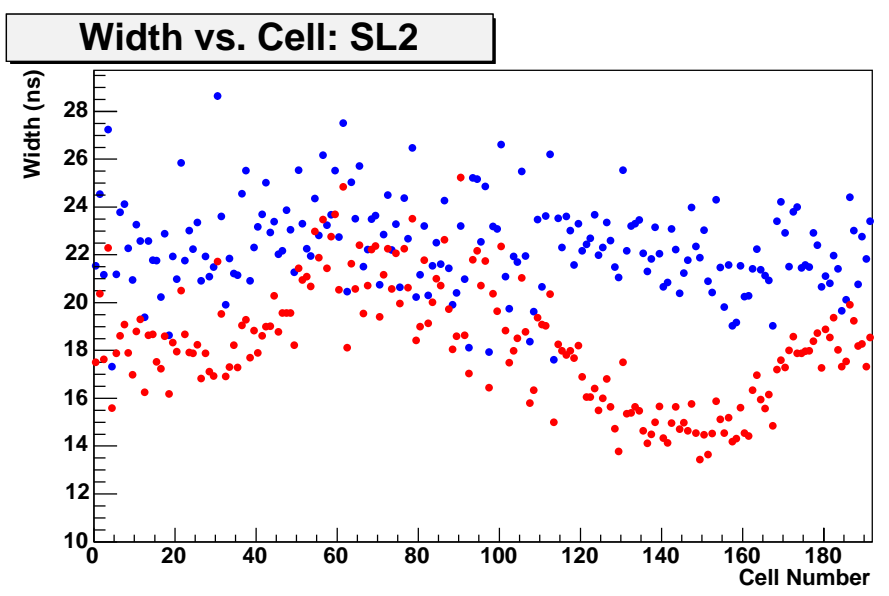

Fig. 2. Width (gain) versus cell number (phi) for SL1. Details are the same as Fig. 1. as a function of $\mathrm{z}$ in the chamber for an early run in August 2002 and for a run taken after most of the aging had occurred in February 2004. The two points in the middle have lower gain because of a wire center support. Gain reduction was also a function of phi as shown in Fig. 2. The region with the largest gain reduction (cells 125-165) is also the coldest. It lies directly under the silicon detector which is kept sub-zero

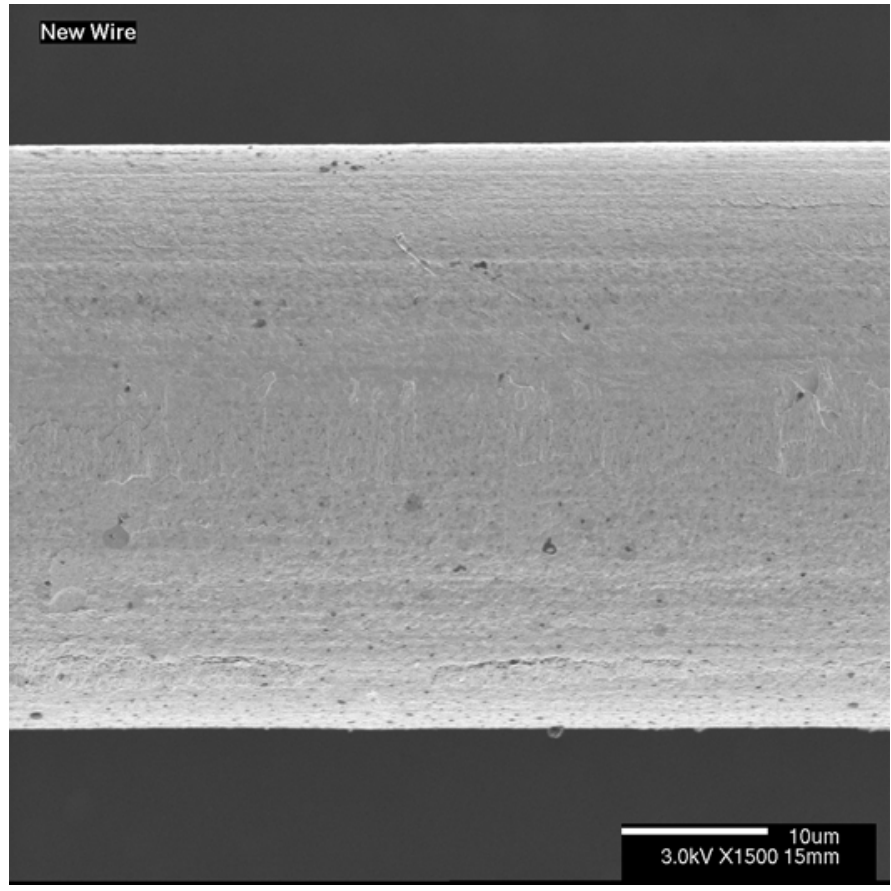

Fig. 3. High resolution SEM picture of a new wire.

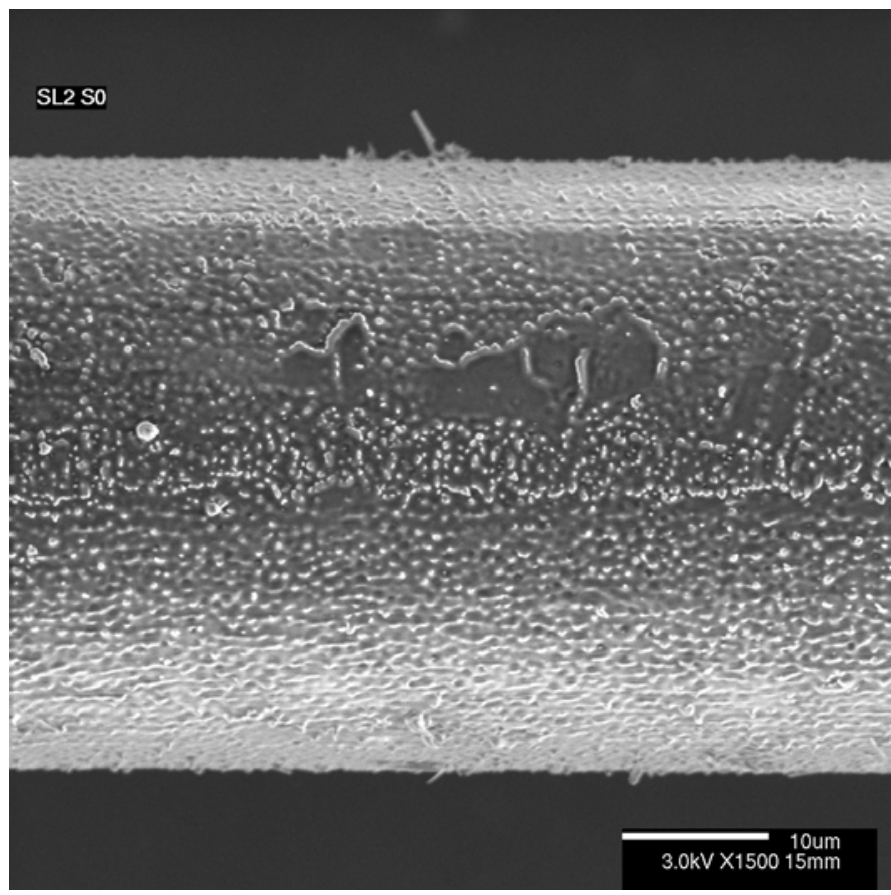

Fig. 4. High resolution SEM picture of an aged wire. The coating is smooth with sub-micron nodules. 
Celsius to minimize the effects of radiation damage. The aging dependence on temperature is consistent with the temperature dependence seen in the $\mathrm{H} 1$ chamber at HERA which uses argon/ethane gas, but with water as an additive instead of alcohol [3]. Convection currents in the gas could also play a role.

In March 2004 an aged wire plane was removed from an inner super-layer of the COT. Scanning Electron Microscope (SEM) measurements were made of a piece of aged wire and compared to a piece of new wire. The surface of the new wire looks brighter and striations are visible (see Fig. 3). The aged wire is dark and smooth except for the sub-micron nodules dotting its surface (see Fig 4).

Electron Dispersive Spectroscopy (EDS) of a new wire showed mostly gold with a little background carbon while the aged wire had a much larger carbon peak and a small oxygen peak (see Fig. 5). EDS uses an electron beam to eject electrons from the inner shells of atoms. The x-ray spectrum generated when these states are refilled gives a measure of the elemental makeup of the sample. It is not sensitive to hydrogen.

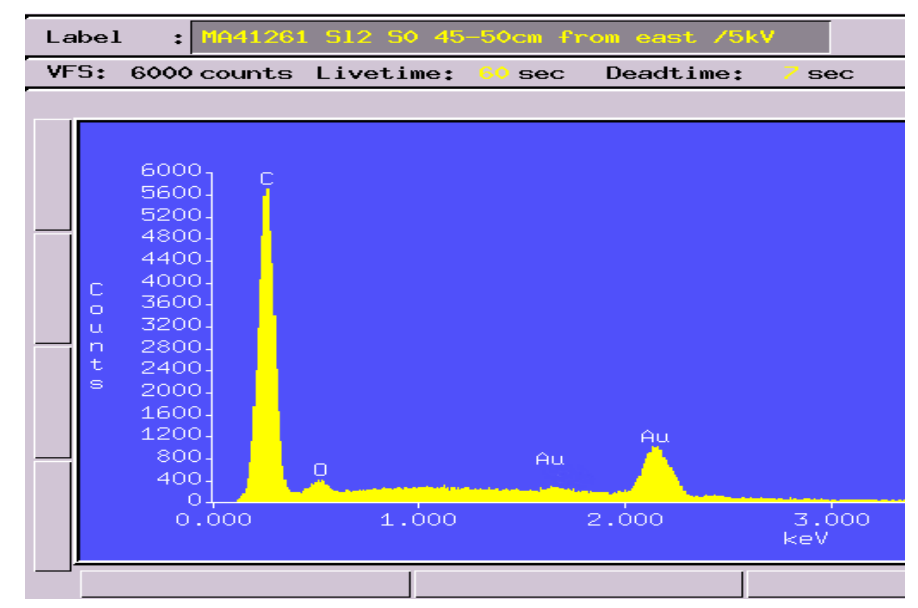

Fig. 5. EDS spectrum of the coating of an aged wire from the COT. A large carbon and small oxygen peak are seen along with gold peaks from the wire.

Some additional measurements were made to study molecular bonds. An X-ray Photoelectron Spectroscopy (XPS) scan indicated primarily $\mathrm{CC}$ and $\mathrm{CH}$ with a few $\mathrm{CO}$ bonds. A Fourier Transform Infrared (FTIR) analysis of the absorption of reflected broadband infrared photons also gives a measure of molecular bonds. It showed a broad absorption band associated with $\mathrm{OH}$ bonds and a cluster of narrow absorption bands associated with $\mathrm{CH}_{2}$ and $\mathrm{CH}_{3}$ bonds.

We found no evidence for aging elsewhere in the COT super-cell. The EDS spectrum of a potential wire in the extracted plane was consistent with that of a new wire. The resistance of the surface of a cathode sheet in that cell was consistent with a new sheet and there was no visible coating. We did not extract a cathode sheet, so it was not possible to perform further microanalysis.

\section{AGING HYPHOTHESIS}

The aging appears to be due to a coating on the sense wires. Presumably polymers, strings made up of groups of $\mathrm{CH}_{2}$, grow in the avalanche environment as the gas moves down the wires. When the strings become long enough, they plate out on the wire. Presumably this "condensation" is temperature dependent. Probably there is also ablation in the avalanches and aging depends on the balance between ablation and deposition. No evidence was found for silicon or other contaminants in the gas that might be causing the aging, although we can't rule out contamination from some aliphatic oil. However, it appears likely that the aging seen in the COT would be present in most hydrocarbon gases. It is a type of aging that is not easy to reproduce in a small chamber with a localized source. The polymers probably have to stay in a radiation environment a long time in order to become copious enough and to grow long enough to make significant deposits on the wires. Also, since it is a multi-step process, the rate of aging (gain decrease) for a wire should increase faster than linearly with respect to the charge collection rate.

In order to combat this sort of aging there are several straightforward steps that can be taken. Several groups have found that increasing the gas flow helps. The purpose is to vent the polymers before they grow long enough to deposit. Increasing the temperature may also help, but often that is difficult because of the proximity of other temperature sensitive detectors. Another standard approach is to add alcohol, methylal, or water. The oxygen in them is supposed to slow the growth of the polymer chains. Some people have suggested adding straight oxygen, but few appear to have done so. An exception is Boyarski [4] who was successful reversing cathode aging by adding 200-500 ppm of oxygen, but not by adding alcohol, methylal, or water. When we finally added oxygen, it also appeared to be very effective in reversing anode aging.

Another popular approach is to get rid of all hydrocarbons in the gas. Switching to $\operatorname{argon} / \mathrm{CO}_{2}$ or $\operatorname{argon} / \mathrm{CF}_{4}$ should eliminate the type of polymerization that we were seeing. However argon $/ \mathrm{CO}_{2}$ is not as forgiving a chamber gas as argon/ethane with respect to chamber breakdown.

\section{REDUCING AGING IN THE COT}

The first step we took was to increase the gas flow into the chamber from the initial $20 \mathrm{SCFH}$ to $40 \mathrm{SCFH}$. The higher flow rate gives a volume exchange every 15 hours.

The next thing we implemented was a gas recirculation system. The initial goal was to achieve a total flow through the chamber of $200 \mathrm{SCFH}$ by recirculating $160 \mathrm{SCFH}$. Cleaning the recirculated gas was left for the future, but we still expected to see an improvement because of better gas flow patterns in the chamber and a dilution of polymers in the inner super-layers. Gas cleanup was expected to be difficult 


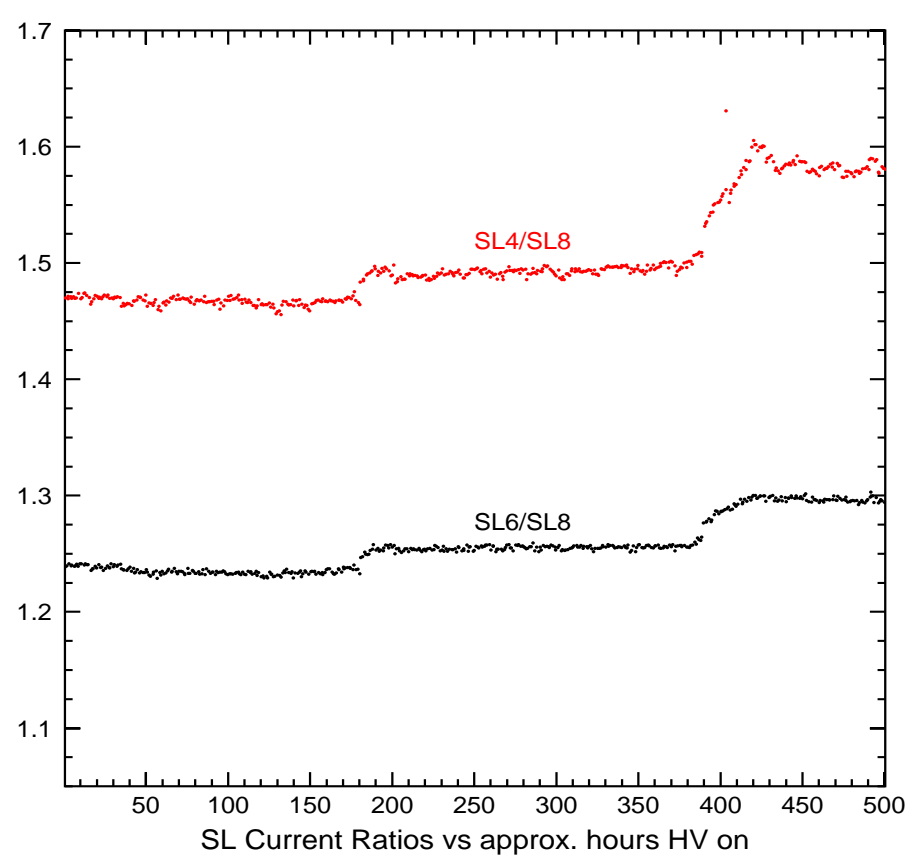

Fig. 6. Currents in SL4 and SL6 normalized to the current in SL8 which had almost no aging. The two jumps in gain correspond to the two times we unintentionally had oxygen in the gas.

because of the large alcohol content that had to be removed from the gas and then added back into the gas.

Since both the peak and integrated luminosity had significant increases during this time period, it was difficult to assess how much these improvements helped. When the input gas rate was increased from 20 to $40 \mathrm{SCFH}$, the apparent aging per unit integrated charge did not appear to change much, but

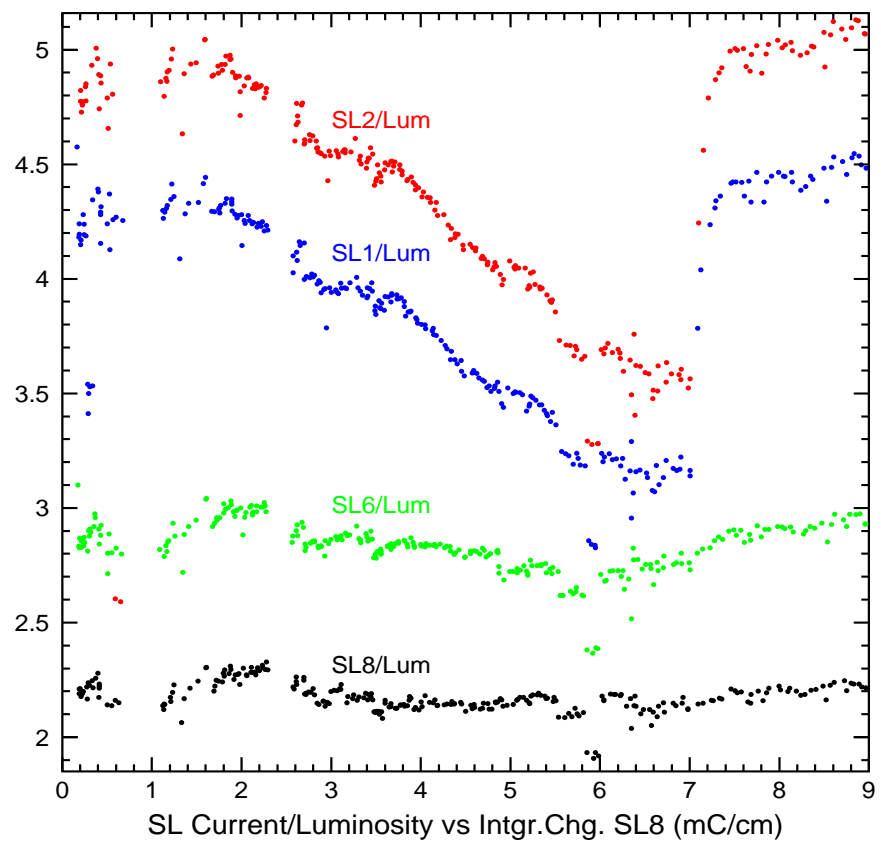

Fig. 7. Super-layer currents normalized to luminosity versus integrated charge for all of Run 2. SL1 is blue, SL2 is red, SL6 is green, and SL8 is black.

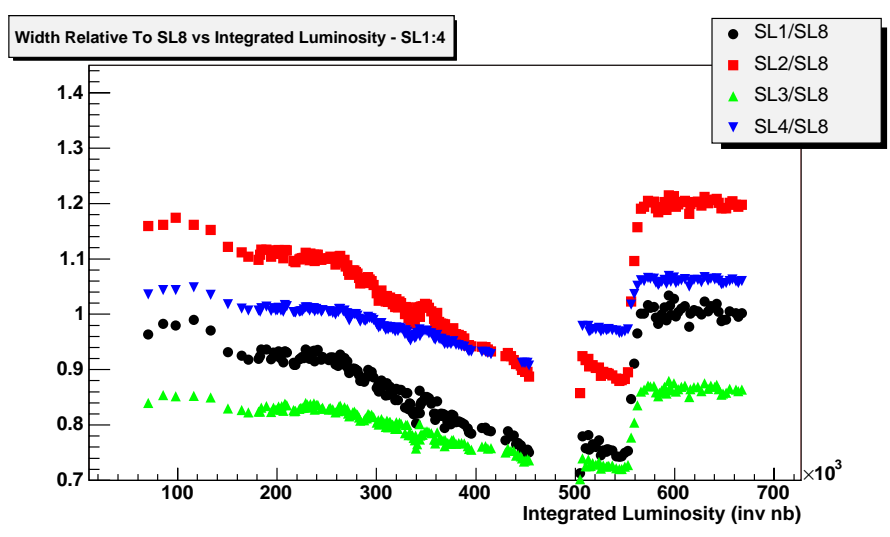

Fig. 8. The average gain (pulse widths) in the inner super-layers normalized to SL8 which experience little aging. SL1 is black, SL2 is red, SL3 is green, and SL4 is blue.

it would have probably increased if we had done nothing because of the higher radiation rates. When we added the recirculation, it appeared to make some improvement, but we did not run long enough to get a definitive measurement.

However, while installing the recirculation system and commissioning it, we unintentionally introduced oxygen (from air leaks) at the $100 \mathrm{ppm}$ level at two well defined points in time. Eventually we made the right plot (see Fig. 6) to be able to correlate the oxygen with gain recovery. Adding oxygen was already on our list of things to try, but we were surprised by the efficacy with which it worked. Under normal operating conditions the oxygen level in the COT was $\leq 12$ ppm; on June 16, 2004 enough air was added to the COT to increase the oxygen level to $\sim 100 \mathrm{ppm}$. Fig. 7 shows the ratios of superlayer current to the instantaneous luminosity for SL1, SL2, SL4, and SL8 plotted against the integrated charge in SL8 for those times when the inner super-layers were active. The intentional introduction of oxygen started at an integrated charge of $7.0 \mathrm{mC} / \mathrm{cm}$ for SL8 (corresponding to $\sim 40 \mathrm{mC} / \mathrm{cm}$ for the inner super-layers). Almost two years of gain loss was reversed in less than 10 days while accumulating $\leq 2 \mathrm{mC} / \mathrm{cm}$ of charge on the inner super-layers. SL8 appears to have suffered little aging and thus can be used as a reference. The other super-layers show varying degrees of gain loss for much of the run. Fig. 8 plots the average width of the inner superlayers normalized to the average width of SL8 versus the integrated luminosity since the beginning of the run. The dependence on many of the systematic effects such pressure, temperature, and gas mixture cancel in the ratios. This plot shows the same features as Fig. 7.

Probably the oxygen inhibits polymer strings from growing by combining with $\mathrm{CH} 2$ radicals or attaching directly to the end of a string [5][6]. Aging presumably depends on the balance between the deposition of polymers and the natural ablation that takes place in an avalanche. In this scenario a small amount of oxygen in the gas inhibits polymerization and ablation dominates. However we can not rule out the possibility that the oxygen is reacting directly with the coating. 


\section{STUDIES TO UNDERSTAND THE AGING}

Several studies and tests were undertaken to help understand the nature of the aging that we were seeing.

One of the first tests we did was to reverse the gas flow in the chamber. There was concern that there might be contamination from one of the endplates. The aging pattern in $\mathrm{z}$ reversed as would be expected if the aging were simply due to polymer buildup from the hydrocarbons in the gas.

Some studies involved looking at the historical data. Based on the HV current data, the gain changed significantly over the first few hours of a store during periods of aging. For this reason, the plots showing the ratio of currents or the ratio of current to luminosity for each store excluded the first 5 hours of the store. There was concern that temperature or charging effects took several hours to stabilize. Fig. 9 effectively shows how the ratio of the gain in the first hour to the gain in the second hour changed for the whole run. During the period of serious aging, the gain was $2-3 \%$ lower during the first hour. After the oxygen was added, the gain drop during the first hour decreased to about $0.4 \%$. Some of this small drop could be a temperature effect since the luminosity has been very high since the oxygen was added. The theory is that by the end of a store polymers have built up both on the wires and in the chamber gas. Between stores the polymers in the gas either deposit on the wires or exit the chamber. When the next store begins, the polymer concentration in the gas is lower so that there is reverse aging for the first couple of hours until the polymer concentration rebuilds. This is consistent with our observation that if the chamber HV was kept at its normal operating point after a store was ended, increased gain loss was seen at the beginning of next store.

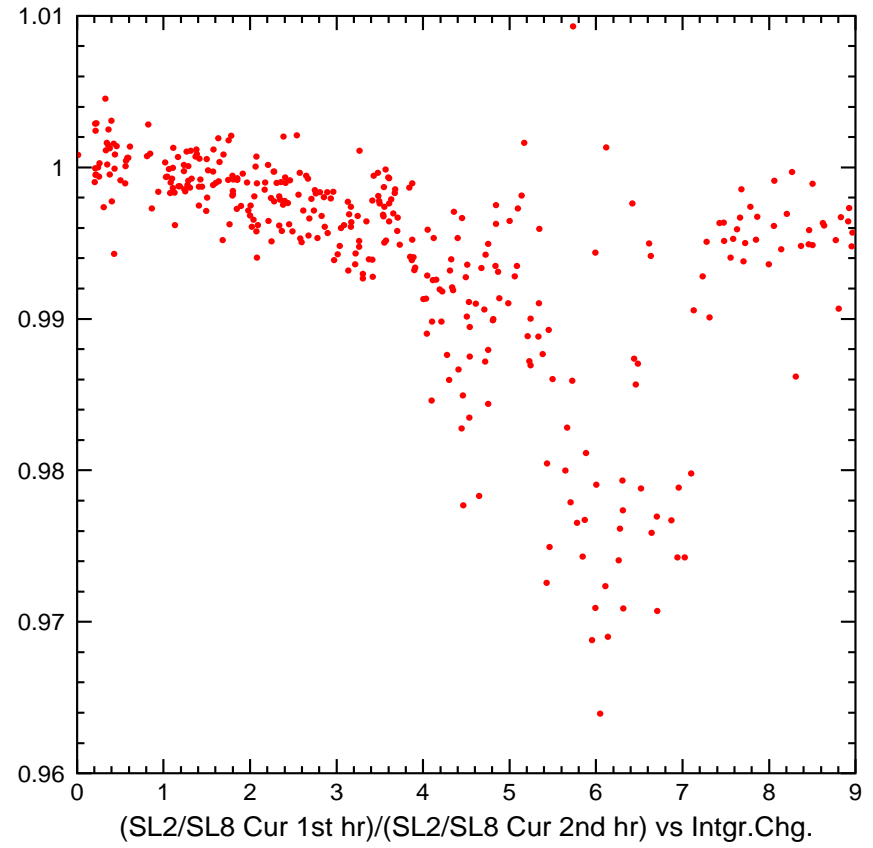

Fig. 9. The dots are the ratio of SL2 gain for the first hour of each store to its gain for the second hour based on HV currents.

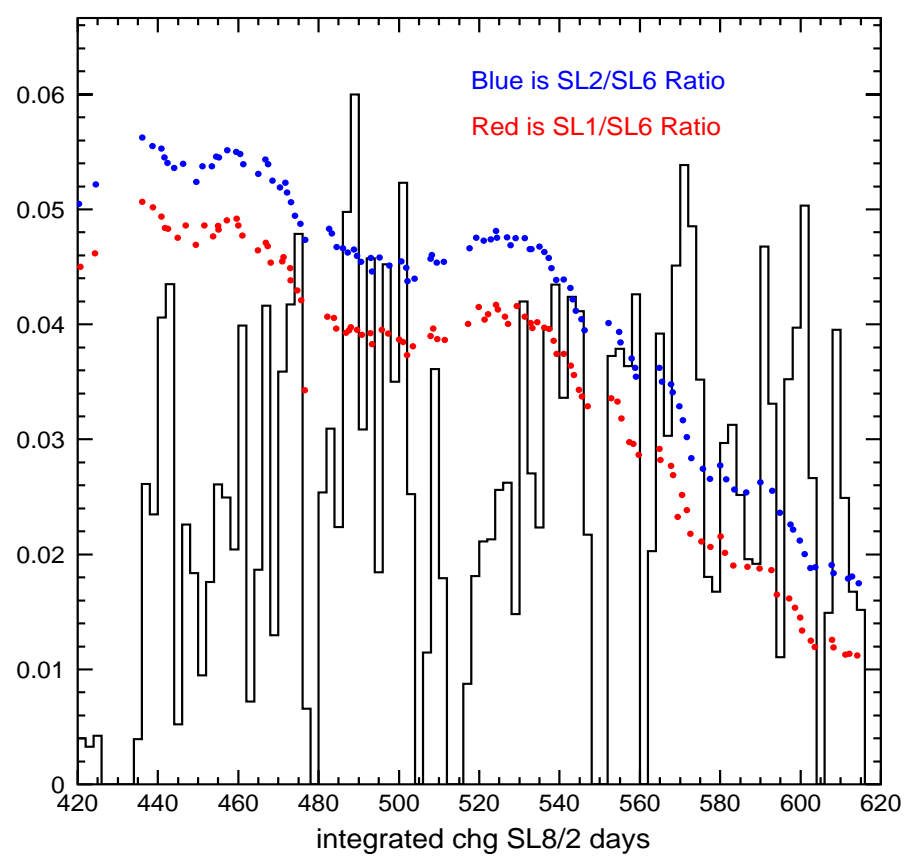

Fig. 10. The black histogram shows the integrated charge per two day period versus the number of days since the beginning of 2002 . Superimposed is the gain (zero suppressed) in SL1 and SL2 calculated from the ratio of their HV currents to those in SL6.

There were times, often just after a shutdown when the accelerator was not working well, that some gain recovery was observed in the chamber. Fig. 10 shows the ratio of the currents in SL1 and SL2 to the current in an outer super-layer superimposed on a histogram of the charge accumulated per two day period. The period starting at 510 days since the beginning of 2002 is one where the inner super-layers recovered relative to the outer super-layers and also one where the integrated charge per unit time was smaller than usual. There was another period starting at day 444 where there was a small recovery which again was associated with less integrated charge than normal.

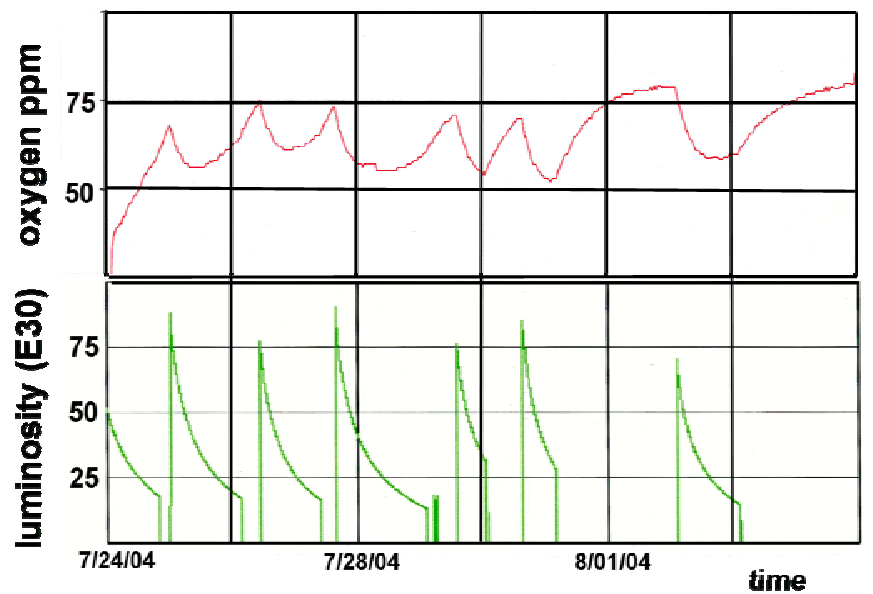

Fig. 11. The upper curve shows the fluctuation in oxygen level and the bottom curve the luminosity for a 10 day period of time. The oxygen level falls when there is beam. 
Another observation was that the oxygen we added appeared to be consumed at a significant rate in the COT during periods of radiation. We add a constant amount of oxygen, but the amount of oxygen observed in the COT exhaust gas decreased by up to $30 \mathrm{ppm}$ depending on the radiation level in the chamber. Fig. 11 shows both the oxygen level and the luminosity for a ten day period starting in late July 2004 (more than a month after the COT gain had been fully recovered). Based on the currents drawn from the HV supplies, about 40 oxygen molecules were lost for each electron collected from the avalanches. This consumption of oxygen is larger than we originally expected and may be due to catalytic oxidation in the presence of the gold wire surface and the avalanches. This consumption rate was confirmed by a second oxygen analyzer from a different manufacturer. It is thought unlikely that there is some product produced in the chamber that would cause a large enough temporary reduction in the sensitivity of the oxygen analyzer to explain our observations. Most sensitivity decreases of this size are permanent. However at this time we cannot completely rule out the possibility that some artifact associated with the oxygen analyzer is causing this effect.

Studies were done on some of the aged wires that were removed from the COT in March. They were put in small chambers with a localized source to see if we could reverse age them. One plane of wire was put in a chamber with the standard COT gas mix of argon/ethane (50/50) with $1.7 \%$
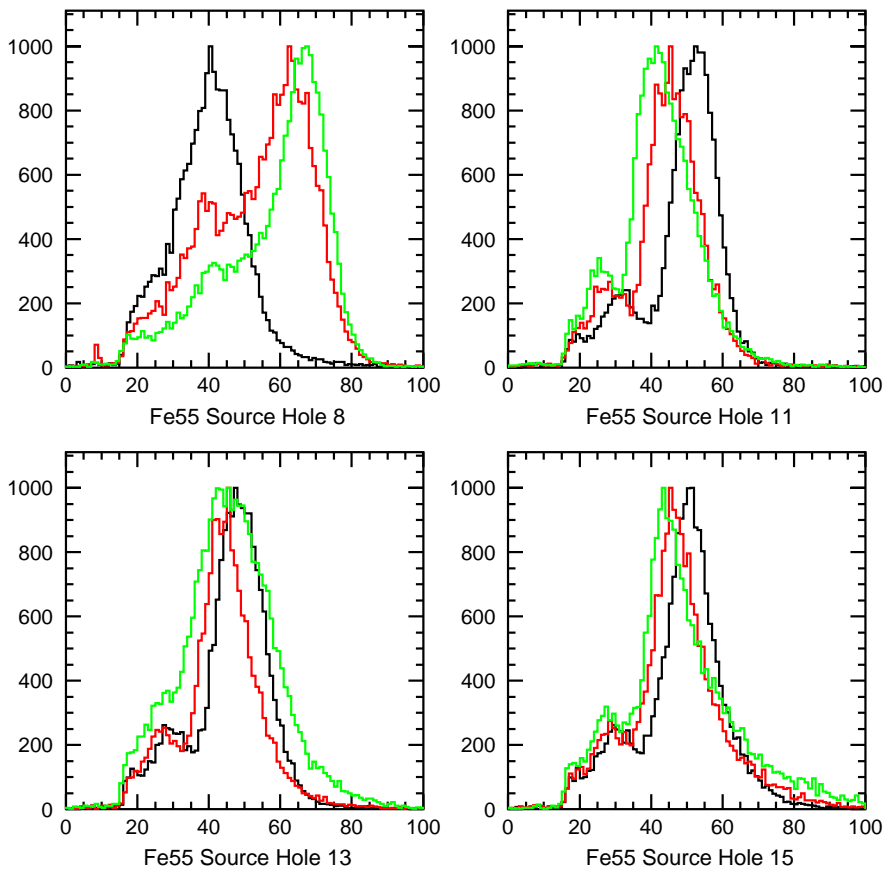

Fig. 12. $\mathrm{Fe}^{55}$ spectra at four different holes in a test chamber constructed with aged wires from the COT and operated with the standard COT gas mix. Hole 8 was irradiated with a $\mathrm{Sr}^{90}$ source in an attempt to recover the gain. The black histogram is the $\mathrm{Fe}^{55}$ spectra before $\mathrm{Sr}^{90}$ irradiation, the red at the midpoint, and the green at the end. Gain was recovered only under the $\mathrm{Sr}^{90}$ source. isopropanol. It was irradiated with a localized $\mathrm{Sr}^{90}$ source and the gain was monitored at several points in the plane by measuring the $\mathrm{Fe}^{55}$ spectrum. It was found the gain made a dramatic recovery underneath the $\mathrm{Sr}^{90}$ source, but it went down elsewhere in the chamber. Fig. 12 shows the $\mathrm{Fe}^{55}$ spectra under hole 8 , the irradiated hole, and three other holes away from the source. When the same test was tried with a gas mixture of argon $/ \mathrm{CO}_{2}$, there was again a dramatic improvement under the $\mathrm{Sr}^{90}$ source, but the gain stayed the same or got slightly better elsewhere in the plane. We also put a single aged wire from the COT in a stainless-steel tube, and exposed it to a similar $\mathrm{Sr}^{90}$ source while operating with exhaust gas from the COT that contained about $100 \mathrm{ppm}$ of oxygen. Again we saw a dramatic gain recovery under the source. If we ran these reverse aging tests long enough, the $\mathrm{Fe}^{55}$ spectrum recovered to normal.

After these reverse aging tests, we viewed the wires with a SEM and performed an EDS analysis of the surface. The wires looked clean and the EDS indicated that most if not all of the coating was removed. One point of note is that the EDS analysis of the wire that had been reverse aged in $\operatorname{argon} / \mathrm{CO}_{2}$ showed an anomalously large amount of oxygen.

An attempt was also made to measure the thickness of the coating on the wires. For $40 \mu \mathrm{m}$ wires we estimate that a $1.0 \mu \mathrm{m}$ conductive coating should reduce the gain by about $20 \%$ and a $2.0 \mu \mathrm{m}$ coating by $40 \%$. From the SEM pictures of places on the wire where part of the coating has been removed, we got estimates of the coating thickness that vary from $0.3 \mu \mathrm{m}$ to $2.0 \mu \mathrm{m}$ (see Fig. 13). The larger thickness is probably enough to explain the gain loss we saw, but a better understanding of the coating thickness and how it is

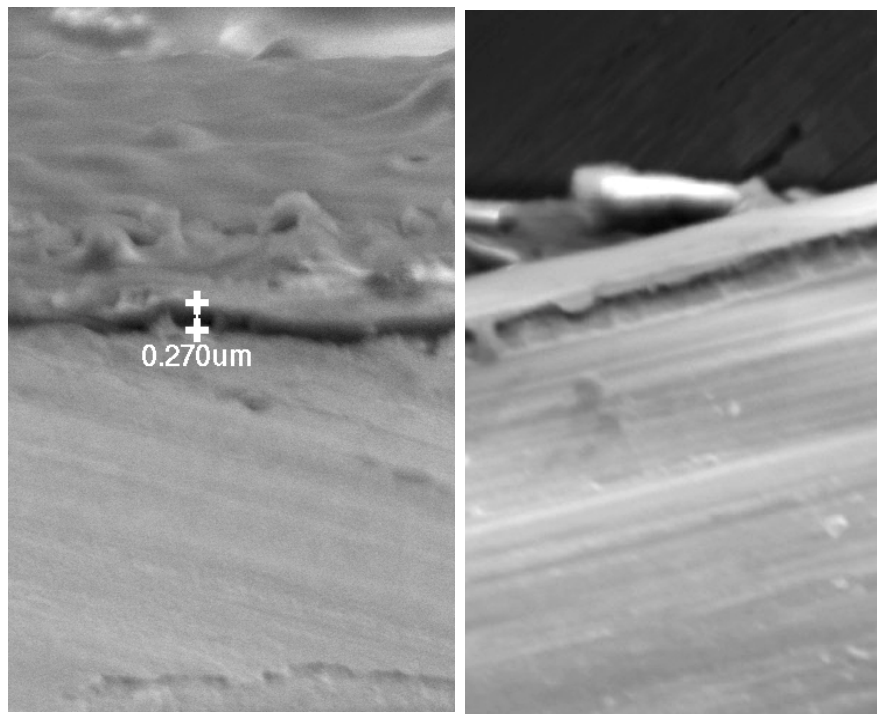

Fig. 13. Two regions where the wire coating is visible in SEM pictures are shown. On the left there is a dark coating that appears to be $0.3 \mu \mathrm{m}$ thick with the wire showing in the foreground. On the right (with different magnification) there appears to be a 1.5-2.0 $\mu \mathrm{m}$ coating on top of a thin light band that is probably the gold plating. Presumably bare tungsten is in the foreground. 
distributed are needed before making a definitive statement. Note that if the resistivity of the coating is greater than $10^{6} \Omega$ $\mathrm{cm}$, then it may have a significant effect on the gain.

\section{CONCLUSIONS}

We found that aging in a clean drift chamber where the primary quenching gas is a hydrocarbon seems to depend on the buildup of polymers in the gas as it flows through the chamber. The deposition of these polymers causes a coating on the wires that reduces the gain. This gain reduction appears to be a multi-step process that depends not only on the integrated charge on the wire, but also on the charge collection rate. The aging also appears to be temperature dependent. Adding a small amount of oxygen, $100 \mathrm{ppm}$ in our case, seems to prevent this type of aging and allows such aging to be reversed. This conclusion does not apply to chambers that have non-hydrocarbon contaminants such as silicon.

The oxygen is consumed in a chemical reaction. Traditionally the oxygen in alcohol or water is supposed to inhibit polymerization by combining with $\mathrm{CH}_{2}$ radicals or attaching to the ends of polymer chains. Our observations taken together with those of Boyarski [4] and Niebuhr [3] suggest that oxygen molecules by themselves are much more efficient at doing this. Aging probably depends on the balance between some natural ablation that occurs in avalanches and the deposition of polymers that have built up in the chamber gas. We can't rule out the possibility that some of the oxygen reacts directly with the wire coating.

These results point out the difficulty of using small test chambers with localized sources to predict aging in larger chambers. They also emphasize the importance of controlling the amount of oxygen in any tests.

\section{ACKNOWLEDGMENT}

The authors would like to thank the Fermilab staff for their vital contributions.

\section{REFERENCES}

[1] R. Blair et al., "The CDF II detector: Technical design report," Fermilab-Pub-96-390-E, 1996.

[2] J. T. Affolder et al., "CDF central outer tracker," Nucl. Instr. Meth., vol. A 526, pp. 249-299, Apr. 2003.

[3] Carsten Niebuhr, "Aging Effects in the Central Jet Chamber of the H1 Experiment," Nucl. Instr. Meth., vol. 515, pp. 43-49, 2003.

[4] A. M. Boyarski, "Additives that prevent or reverse cathode aging in drift chambers with helium-isobutane gas," Nucl. Instr. Meth., vol. A 515, pp. 190-195, 2003.

[5] J. Va'Vra, "Physics and chemistry of aging - early developments," Nucl. Instr. Meth., vol. A 515, pp. 1-14, 2003.

[6] Hirotsugu Yasuda, "New Insights into aging phenomena from plasma chemistry," Nucl. Instr. Meth., vol. A 515, pp. 15-30, 2003. 